\title{
A Search for the Regional Variations in the Wavelength Dependence of Interstellar Polarization
}

\author{
K. SERKowsKi, W. ChojNACKI, AND S. RUCINSKI \\ Warsaw University Observatory \\ Warsaw, Poland
}

TTHE POLARIZATION OF 19 BRIGHT, HIGHLY POLARIZED STARS was 1 measured from March until July 1965, with a differential polarimeter installed at the Cassegrain focus of the $90-\mathrm{cm}$ telescope of the Torun Observatory in Poland. The stellar light was split by the calcite Wollaston prism into two beams falling upon the cathodes of the photomultipliers. The observations were made at eight position angles of the polarimeter differing by $45^{\circ}$, alternately with and without the quartz depolarizer. The yellow 2-mm Schott GG 14 and the blue Schott BG 12 (1-mm)+ GG 13 (2-mm) filters were used. No optical elements except the depolarizer were placed between the Wollaston prism and the telescope mirrors.

Usually only the Stokes parameters having the larger absolute values were accurately measured. The resulting polarization ratios $p_{V} / p_{B}$ of the Stokes parameters expressed in magnitudes, measured with yellow and blue filters, are given in the ninth column of table I. They are followed by the number of nights that the star was observed; each observation lasted about an hour. Several nearby stars from the list published in reference 1 were also observed; these observations indicate that the instrumental polarization is negligibly small.

Table I contains all the stars for which the ratio of the amounts of polarization in yellow and blue spectral regions is known with a mean error not larger than $\pm \mathbf{0 . 0 5}$. It includes the observations reported in references 2 to 5 , and those made at the Torun Observatory. The results of these references are followed in table $I$ by their mean errors. The value $p_{4290}$ is the average of the amounts of polarization measured by Treanor (ref. 4) at $4050 \AA$ and $4530 \AA$, while $p_{5250}$ is the average of those at $5000 \AA$ and $5500 \AA$. In the last column of table I the arithmetic mean of the polarization ratios listed in preceding columns is given for each star. No appreciable systematic differences between the results of different authors are found. 
TABLE I. - Ratios of Interstellar Polarization in

\begin{tabular}{|c|c|c|c|c|c|}
\hline $\begin{array}{l}\text { HD or cluster } \\
\text { number }\end{array}$ & Star & $\begin{array}{c}111 \\
\operatorname{deg}\end{array}$ & $\frac{E_{V-1}}{E_{V-A}}$ & $\begin{array}{c}\frac{p_{5160}}{p_{4300}} \\
\text { (ref. 2) }\end{array}$ & $\begin{array}{l}\frac{p_{5580}}{p_{4200}} \\
\text { (ref. 3) }\end{array}$ \\
\hline 147165 & $\sigma$ Sco & 351 & 2.20 & & \\
\hline 147933 & $\rho \mathrm{Oph}$ & 354 & 2.14 & & \\
\hline 160529 & & 356 & ......... & & \\
\hline 149757 & $\zeta \mathrm{Oph}$ & 6 & $2.00:$ & $1.09 \pm .03$ & \\
\hline M 25 & 77 stars & 14 & & & \\
\hline 161056 & & 18 & $\cdots$ & & $1.02 \pm .02$ \\
\hline 154445 & & 19 & ......... & .................. & $1.08 \pm .01$ \\
\hline 183143 & & 53 & 1.89 & 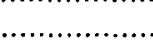 & $1.07 \pm .01$ \\
\hline NGC 6823 & 16 stars & 59 & 2.11 & & ................ \\
\hline 193237 & P Cyg & 76 & 1.75: & & \\
\hline 194279 & & 78 & 2.00 & & \\
\hline VI Cyg & 27 stars & 80 & 1.80 & & \\
\hline 194057 & & 81 & & & ................. \\
\hline 198478 & 55 Cyg & 86 & 1.91 & & $1.00 \pm .02$ \\
\hline 197770 & & 93 & & & \\
\hline 204827 & & 99 & & & \\
\hline 207260 & $\nu$ Cep & 102 & 1.82 & & \\
\hline 213470 & & 104 & ......... & & \\
\hline 217476 & & 108 & 1.76 & & \\
\hline 5394 & $\gamma$ Cas & 123 & $1.86:$ & $1.11 \pm .05$ & \\
\hline 6811 & $\varphi$ And & 126 & 1.7: & $0.88 \pm .05$ & \\
\hline 7927 & $\varphi$ Cas & 127 & 1.98 & & \\
\hline 10516 & $\varphi$ Per & 131 & 1.86: & $.92 \pm .04$ & \\
\hline 13402 & & 133 & & & \\
\hline$+58^{\circ} 400$ & & 133 & ............ & & \\
\hline Stock 2 & 10 stars & 133 & ........... & & \\
\hline 17378 & & 138 & 1.90 & & ............. \\
\hline 21291 & 2H Cam & 141 & 2.15 & & $1.00 \pm .02$ \\
\hline 21389 & & 142 & 1.96 & & \\
\hline 25443 & & 143 & & & \\
\hline 24912 & $\xi$ Per & 160 & 1.94 & $1.16 \pm .05$ & \\
\hline 24398 & $\zeta$ Per & 162 & 2.17: & $1.04 \pm .02$ & \\
\hline NGC 1893 & 19 stars & 174 & ........ & & \\
\hline 43384 & $9 \mathrm{Gem}$ & 188 & 2.08 & & \\
\hline 41117 & $\chi^{2}$ Ori & 189 & 2.00 & & \\
\hline 37356 & near $M 42$ & 208 & & & \\
\hline
\end{tabular}

${ }^{a}$ Value for $p_{5250} / p_{4530}$. 
Yellow and Blue Spectral Regions

\begin{tabular}{|c|c|c|c|c|}
\hline $\begin{array}{c}\frac{p_{5250}}{p_{4290}} \\
(\text { ref. 4) }\end{array}$ & $\begin{array}{c}p_{v} / p_{B} \\
\text { (ref. 5) }\end{array}$ & $\begin{array}{c}p_{v} / p_{B} \\
\text { (present } \\
\text { paper) }\end{array}$ & $\begin{array}{l}\text { Number } \\
\text { of nights } \\
\text { observed } \\
\text { (present } \\
\text { paper) }\end{array}$ & $\begin{array}{c}\text { Mean } \\
\text { value of } \\
p_{v} / p_{\theta}\end{array}$ \\
\hline $1.13 \pm .04$ & & & & 1.13 \\
\hline $1.11 \pm .04$ & ............... & & & 1.11 \\
\hline a $1.10 \pm .06$ & $1.03 \pm .02$ & & & 1.06 \\
\hline $1.08 \pm .04$ & & & & 1.08 \\
\hline & $1.03 \pm .02$ & & ........... & 1.03 \\
\hline $1.13 \pm .05$ & $1.13 \pm .05$ & $1.11 \pm .03$ & 2 & 1.10 \\
\hline $0.98 \pm .04$ & $1.19 \pm .02$ & $1.06 \pm .02$ & 4 & 1.08 \\
\hline $1.10 \pm .02$ & $1.07 \pm .02$ & $1.09 \pm .02$ & 2 & 1.08 \\
\hline & $\begin{array}{c}1.08 \pm .02 \\
\ldots \ldots \ldots \ldots \ldots\end{array}$ & $0.93 \pm .05$ & 2 & $\begin{array}{r}1.08 \\
.93\end{array}$ \\
\hline & …………....... & $1.04 \pm .03$ & 2 & 1.04 \\
\hline & $0.93 \pm .02$ & & & .93 \\
\hline & & $1.01 \pm .04$ & 2 & 1.01 \\
\hline $0.95 \pm .02$ & $1.00 \pm .04$ & $1.02 \pm .03$ & 3 & .99 \\
\hline & $\ldots, \ldots \ldots \ldots \ldots$ & $1.01 \pm .03$ & 3 & 1.01 \\
\hline & …............ & $0.97 \pm .02$ & 5 & .97 \\
\hline & & $0.96 \pm .04$ & 2 & .96 \\
\hline & & $1.02 \pm .04$ & 2 & 1.02 \\
\hline $0.97 \pm .03$ & (n., & $0.98 \pm .08$ & 2 & .97 \\
\hline & & & & 1.11 \\
\hline & & & & .88 \\
\hline & $1.03 \pm .04$ & $1.02 \pm .02$ & 2 & 1.02 \\
\hline & ................ & & & .92 \\
\hline & $1.03 \pm .05$ & & & 1.03 \\
\hline & $1.02 \pm .03$ & & ... & 1.02 \\
\hline & $1.09 \pm .04$ & 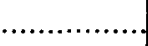 & $\ldots$ & 1.09 \\
\hline & ................. & $1.06 \pm .04$ & 1 & 1.06 \\
\hline & $1.01 \pm .02$ & …............ & .... & 1.00 \\
\hline & ................. & $1.00 \pm .05$ & 1 & 1.00 \\
\hline & & $0.99 \pm .03$ & 1 & .99 \\
\hline & & & & 1.16 \\
\hline & & & & 1.04 \\
\hline ................. & $1.03 \pm .04$ & .................... & $\cdots$ & 1.03 \\
\hline & $1.08 \pm .02$ & $1.03 \pm .03$ & 3 & 1.06 \\
\hline & & $1.09 \pm .05$ & 1 & 1.09 \\
\hline$\ldots$ & & $1.11 \pm .05$ & 4 & 1.11 \\
\hline
\end{tabular}




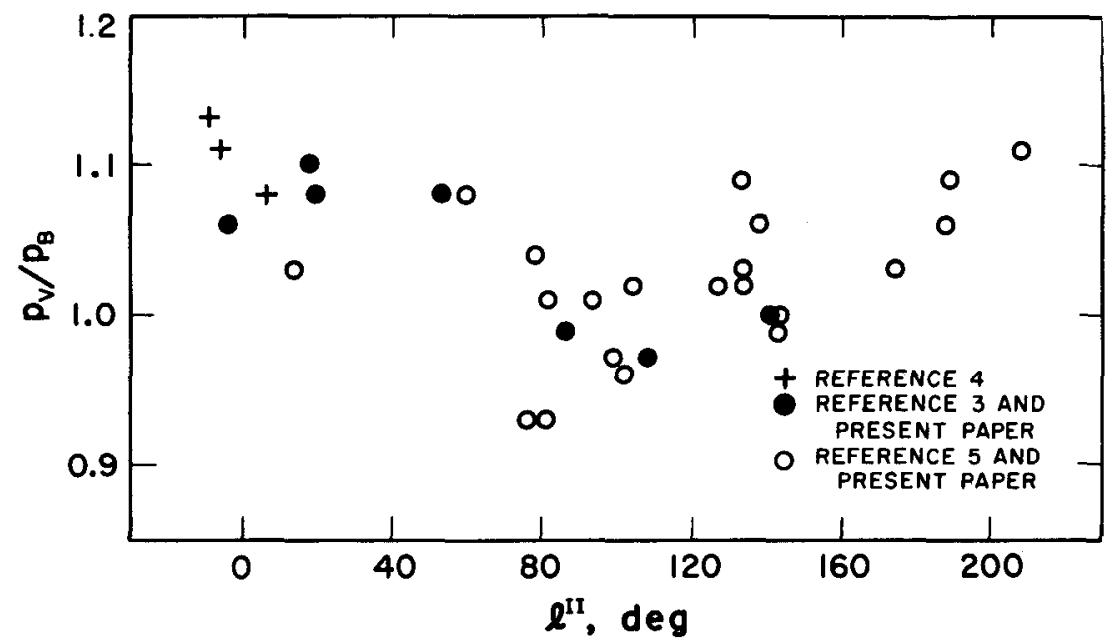

FIGURE 1. - Ratio of amount of polarization in yellow to amount in blue spectral region as a function of galactic longitude of the star.

The dependence of the mean ratios of yellow and blue amounts of polarization as a function of galactic longitude is shown in figure 1 . The smallest values of this ratio occur in the Cygnus region.

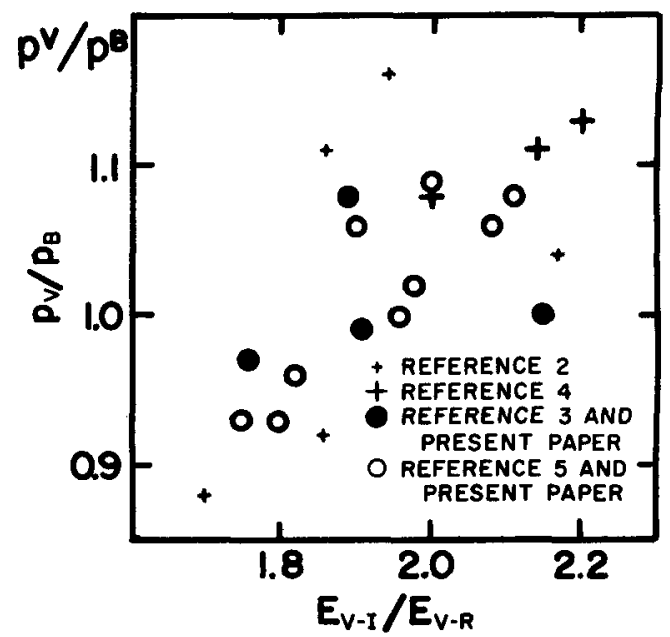

Figure 2. - Ratio of amount of polarization in yellow to amount in blue spectral region as a function of ratio of the color excesses $E_{V-I} / E_{V-R}$.

The ratio of polarizations is correlated with the color excesses ratio $E_{V-I} / E_{V-R}$ as obtained in reference 6 in figure $2 .{ }^{1}$ The ratios of the color

\footnotetext{
" Note added in proof: Recent observations of the wavelength dependence of interstellar polarization by G. V. Coyne and T. Gehrels (Astron. J., vol. 71, p. 355, 1966) and by K. Serkowski (unpublished) confirm the validity of this correlation.
} 
excesses listed in table $I$ and plotted in figure 2 are computed by using the observed values of $V-R$ and $V-I$ and intrinsic colors listed in reference 6,7 , or 8 . In order to bring into agreement the results of these papers, the corrections 0.09 and 0.05 were added to the $V-R$ and $V-I$ colors observed by the authors of reference 8. For the Orion Sword region, the ratio $E_{V-l} / E_{V-R}$ equals about 2.04 and is only slightly larger than the average for all the sky; similarly the ratio $p_{V} / p_{B}$ is 1.11 for the star HD 37356 situated near the Orion trapezium. The ratio $E_{V-I} / E_{V-R}$ is independent of galactic longitude for those stars in the catalogues of references 6 and 8 for which the amount of polarization is larger than 0.020 magnitude.

The polarimeter described previously was also used at the HauteProvence Observatory in France for attempts to measure the circularly polarized component of stellar light in the yellow spectral region. The values obtained for the Stokes parameter $p_{v}$ describing the circularly polarized component of stellar light expressed in magnitudes are listed with their mean errors in table II. They are accompanied by the amount of polarization $p$ and the position angle of the plane of vibration $\theta$ observed in the yellow spectral region with the same instrument used for the stars with measured $p_{v}$. For none of these stars does $p_{v}$ exceed 0.0004 magnitude, an indication that the degree of ellipticity does not exceed 0.02 percent. This may be considered as an argument against the interstellar grains with high iron content, as these are expected to introduce an appreciable circularly polarized component into the partially plane-polarized stellar light. (See ref. 1.)

TABLE II.-Observations of the Circularly Polarized Component of Stellar Light

\begin{tabular}{|c|l|r|r|r|}
\hline $\begin{array}{c}\text { HD } \\
\text { number }\end{array}$ & Star & $p$ & $\theta, \mathrm{deg}$ & $p_{v}$, magnitude \\
\hline & & & & \\
21291 & $2 \mathrm{H} \mathrm{Cam}$ & 0.073 & 116 & $+0.0004 \pm .0003$ \\
36371 & $\chi$ Aur & .048 & 176 & $-.0002 \pm .0002$ \\
109358 & $\beta$ CVn & .001 & 177 & $.0000 \pm .0002$ \\
112185 & $\epsilon$ UMa & .000 & $\ldots \ldots \ldots \ldots .$. & $-.0001 \pm .0008$ \\
$144217-8$ & $\beta$ Sco & .017 & 91 & $+.0003 \pm .0002$ \\
147165 & $\sigma$ Sco & .032 & 179 & $-.0001 \pm .0006$ \\
149757 & $\zeta$ Oph & .030 & 126 & $+0001 \pm .0003$ \\
174638 & $\beta$ Lyr & .016 & 156 & $+.0001 \pm .0003$ \\
198478 & $55 \mathrm{Cyg}$ & .061 & 3 & $-.0001 \pm .0003$ \\
\hline
\end{tabular}




\section{REFERENCES}

1. Serkowski, K.: Suggested Standards of Polarization. Lowell Obs. Bull., vol. 4, 1960, p. 317 .

2. BEHR, A.: Beobachtungen zur Wellenlängenabhängigkeit der Interstellaren Polarisation. Zs. Astrophys., vol. 47, 1959, p. 54.

3. Gehrels, T.: The Wavelength Dependence of Polarization II. Interstellar Polarization, Astron. J., vol. 65, 1960, p. 470.

4. Treanor, P. J.: Wavelength Dependence of Interstellar Polarization. Astron. J., vol. 68,1963, p. 185.

5. Serkowski, K.: Polarization of Galactic Clusters M25, NGC 869, 884, 1893, Z422, 6823, 6871, and Association VI Cygni. Astrophys. J., vol. 141, 1965, p. 1340.

6. Johnson, H. L.: Interstellar Extinction in the Galaxy. Astrophys. J., vol. 141, 1965, p. 923.

7. Iriarte, B.; Johnson, H. L.; Mitchell, R. I.; and Wisniewski, W. Z.: Five-Color Photometry of Bright Stars. Sky and Telescope, vol. 30, 1965, p. 21.

8. Johnson, H. L.; and Borgman, J.: The Law of Interstellar Extinction. Bull. Astron. Inst. Netherlands, vol. 17, 1963, p. 115. 